\title{
Biosimilar Pegfilgrastim-cbqv Demonstrated Similar Immunogenicity to Pegfilgrastim in Healthy Subjects Across Three Randomized Clinical Studies
}

\author{
Francesca Civoli (D) - Barbara Finck (D) - Helen Tang · Jennifer Hodge • \\ Hillary O’Kelly · Vladimir Vexler
}

Received: November 13, 2021 / Accepted: December 14, 2021 / Published online: January 16, 2022

(C) The Author(s) 2022

\begin{abstract}
Introduction: Biologic therapeutics can trigger immune responses in patients. As part of the totality of evidence that is required for regulatory approval of biosimilars, immunogenicity similarity must be assessed in the clinical programs. Pegfilgrastim-cbqv $\left(\mathrm{UDENYCA}^{\circledR}\right.$ ) is a pegfilgrastim biosimilar approved in the USA and European Union. This article demonstrates the similar immunogenicity of pegfilgrastimcbqv compared with its reference product, pegfilgrastim (Neulasta ${ }^{\circledR}$ ).

Methods: The immunogenicity of pegfilgrastimcbqv was assessed in three clinical studies in healthy subjects (one specifically designed to evaluate immunogenicity similarity and two studies to assess pharmacokinetics and pharmacodynamics bioequivalence) using a tiered approach, in which plasma samples were tested for the presence of antidrug antibodies (ADAs) as
\end{abstract}

Supplementary Information The online version contains supplementary material available at https:// doi.org/10.1007/s12325-021-02024-x.

F. Civoli $(\bowtie) \cdot$ B. Finck $\cdot$ H. Tang $\cdot$ J. Hodge

H. O'Kelly · V. Vexler

Coherus BioSciences, Inc., 333 Twin Dolphin Drive

Suite 600, Redwood City, CA 94065, USA

e-mail: fcivoli@coherus.com

Present Address:

J. Hodge

Myovant Sciences Inc., Brisbane, CA, USA well as ADA binding-specificity, titer and neutralizing activity. To assess the clinical impact of ADAs, pharmacokinetics, pharmacodynamics and safety profiles were compared between ADApositive and -negative subjects.

Results: These studies demonstrated similar immunogenicity of pegfilgrastim-cbqv and pegfilgrastim. The small differences in ADA incidence between treatment groups observed in the immunogenicity study were driven by non-neutralizing, low-titer, polyethylene glycol (PEG)-reactive ADAs, which are commonly present in healthy subjects. No treatmentemergent neutralizing antibodies (NAbs) were detected in either treatment group, and there was no apparent impact of ADAs on pharmacokinetics, pharmacodynamics or safety.

Conclusion: Pegfilgrastim-cbqv has similar immunogenicity to pegfilgrastim. The presented immunogenicity, pharmacokinetics, pharmacodynamics and safety data support the overall demonstration of no clinically meaningful differences between pegfilgrastim-cbqv and pegfilgrastim.

Clinical Trial Registration: NCT02418104 (CHS-1701-04, April 2015), NCT02650973 (CHS1701-05, February 2016) and NCT02385851 (CHS-1701-03, March 2015).

Keywords: UDENYCA; Pegfilgrastim; G-CSF; Immunogenicity; Febrile neutropenia; Biosimilar 
Key Summary Points

This study demonstrates that the immunogenicities of pegfilgrastim-cbqv and its reference product pegfilgrastim are highly similar

Evaluation of pharmacokinetics, pharmacodynamics and safety in antidrug antibody (ADA)-positive and -negative subjects showed that anti-pegfilgrastim antibodies had no clinically meaningful impact

This article demonstrates the low immunogenic risk of pegfilgrastim-cbqv and supports the overall demonstration of similarity between pegfilgrastim-cbqv and pegfilgrastim

\section{INTRODUCTION}

Biosimilars are biologic therapeutics that are highly similar in structure, function, efficacy and safety to their reference product, the licensed biologic therapeutic against which a biosimilar candidate is evaluated [1-4]. A biosimilar undergoes an extensive review process to confirm that there are no clinically meaningful differences in safety, purity and potency compared with its reference product $[1-3,5]$. Both the US Food and Drug Administration (FDA) and European Medicines Agency (EMA) require a totalityof-evidence approach to establish similar efficacy and safety, which includes demonstration of analytical (structural and functional) similarity and clinical demonstration of similar pharmacokinetics (PK), efficacy, safety and immunogenicity $[1,2]$. All biologic therapeutics can be immunogenic or elicit an immune response. The clinical impact of the immune response, measured as circulating antidrug antibodies (ADAs), varies from no clinical impact to reduced or no efficacy to severe adverse events (AEs).

Pegfilgrastim (Neulasta ${ }^{\circledR}$; Amgen, Thousand Oaks, CA) is a pegylated, long-acting form of filgrastim (granulocyte colony-stimulating factor $[\mathrm{G}-\mathrm{CSF}])[6,7]$. In contrast to filgrastim, pegfilgrastim is not prematurely eliminated from circulation by the kidney but is cleared by binding to the G-CSF receptor and subsequent internalization by neutrophils and their precursor cells $[8,9]$. Thus, the addition of polyethylene glycol (PEG) allows for slower clearance and results in less frequent injections for patients $[8,9]$. Pegfilgrastim-cbqv (UDE$\mathrm{NYCA}^{\circledR}$; Coherus BioSciences, Redwood City, CA) is a biosimilar of pegfilgrastim, approved by the FDA and EMA for the prophylaxis of febrile neutropenia in patients receiving myelosuppressive chemotherapy $[7,10]$. The immunogenicity of pegfilgrastim is low with no reported clinical impact [7]; however, in theory an immune response against pegfilgrastim could neutralize its activity or accelerate drug clearance and thus reduce its efficacy. Although an immune response against the polyethylene glycol (PEG) portion of the pegfilgrastim molecule is not expected to have safety consequences, an ADA binding the molecule's G-CSF portion might also neutralize endogenous G-CSF, with potential adverse safety consequences. Therefore, a thorough assessment and characterization of the immunogenicity of pegfilgrastim-cbqv and pegfilgrastim were undertaken as part of the clinical development program to demonstrate that there were no clinically meaningful differences.

Here we present clinical data evaluating the immunogenicity of pegfilgrastim-cbqv compared with its reference product, pegfilgrastim. These data were generated in healthy, immunecompetent subjects, as these individuals comprise the most sensitive population in which to evaluate potentially subtle differences in immunogenicity [4]. Data from the key immunogenicity study (CHS-1701-04) along with a pooled analysis of immunogenicity data from CHS-1701-04 and two single-dose, crossover PK/pharmacodynamics (PD) studies in healthy subjects (CHS-1701-05 and CHS-170103) are presented. The pooled analysis was conducted to increase both the size of the dataset and the robustness of the immunogenicity data comparing pegfilgrastim-cbqv with pegfilgrastim. 


\section{METHODS}

\section{Study Designs and Objectives}

The immunogenicity of pegfilgrastim-cbqv was assessed in clinical studies CHS-1701-04, CHS1701-05 and CHS-1701-03 (Fig. 1). The same dose $(6 \mathrm{mg}$ pegfilgrastim or pegfilgrastim-cbqv in $0.6 \mathrm{ml}$ deliverable volume) was administered in all three studies. The studies were conducted in accordance with all laws and regulations applicable at the respective study sites, and they complied with the International Conference for Harmonization E6 Guideline on Good Clinical Practice and the Declaration of Helsinki. The protocols and informed consent forms were approved by the study sites' institutional review boards (IRBs) before initiation of the studies.

Study CHS-1701-04 (NCT02418104) was a randomized, double-blind, two-period, parallel- arm study designed to assess the immunogenicity (primary objective) and safety of two sequential doses of pegfilgrastim-cbqv compared with pegfilgrastim in healthy subjects. Preexisting (predose at baseline) and treatmentemergent (negative at baseline and positive after dose) ADAs and neutralizing antibodies (NAbs) were summarized by treatment group. Immunogenicity outcomes were: (1) the percentage of subjects with treatment-emergent, titer $\geq 2$, persistent (defined as $\geq 2$ positive time points with $\geq 1$ positive time point after second dose) ADAs and (2) the percentage of subjects with NAbs. Secondary objectives were the assessment of the impact of ADAs and NAbs on the PK, PD and safety profile of pegfilgrastim-cbqv. Patients were randomly assigned 1:1 to treatment groups with study site as the stratification factor. A total of 303 subjects were randomized and treated. Three hundred three

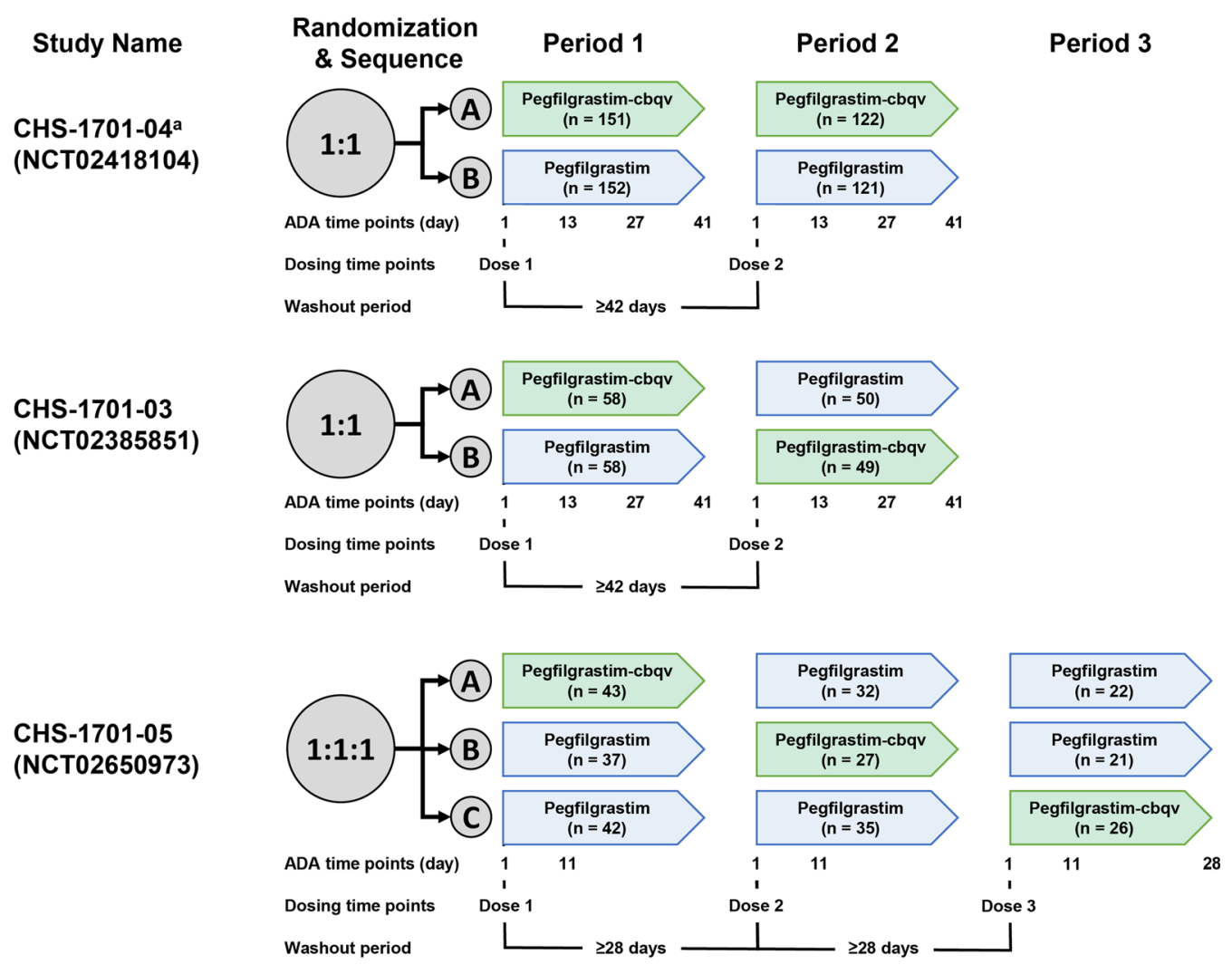

Fig. 1 Study schematics. an study CHS-1701-04, 303 subjects were randomized and evaluated for safety. Due to protocol deviation (some subjects receiving the incorrect dose 2), a total of 268 subjects were included in the ADA analyses. $A D A$ antidrug antibody 
subjects were randomized into the study: 151 subjects to receive pegfilgrastim-cbqv and 152 subjects to receive pegfilgrastim. IRB approval was obtained from Chesapeake IRB (currently Advarra; Columbia, MD, USA), IntegReview Independent IRB (Austin, TX, USA), Midlands IRB (Overland Park, KS, USA) and Western IRB (WIRB, Puyallup, WA, USA).

Study CHS-1701-05 (NCT02650973) has been previously described [11]. Briefly, this study was a randomized, single-blind, partial reference-replicated, three-sequence, three-period crossover study to assess PK and PD bioequivalence of pegfilgrastim-cbqv and pegfilgrastim in healthy subjects. Patients were randomly assigned 1:1:1 to treatment sequence groups with study site and sex as stratification factors. Each treatment sequence group (A, B, or $C$ ) included one dose of pegfilgrastim-cbqv and two doses of pegfilgrastim. One hundred twenty-two subjects were randomized to one of three treatment sequences; each included one dose of pegfilgrastim-cbqv and two doses of pegfilgrastim separated by $\geq 28$ days. IRB approval was obtained from Schulman IRB (Cincinnati, OH, USA), Alpha IRB (San Clemente, CA, USA), Chesapeake IRB and IntegReview Independent IRB.

Study CHS-1701-03 (NCT02385851) was a randomized, double-blind, single-dose, twoperiod crossover study to assess $\mathrm{PK}$ and $\mathrm{PD}$ bioequivalence of a single dose of pegfilgrastim-cbqv versus a single dose of pegfilgrastim in healthy subjects. One hundred sixteen healthy volunteer subjects were screened and randomized into the study: 58 each into Treatment Sequence A (pegfilgrastim-cbqv followed by pegfilgrastim) and Treatment Sequence B (pegfilgrastim followed by pegfilgrastim-cbqv). IRB approval was obtained from IntegReview Independent IRB.

\section{Study Population}

All three studies enrolled adults aged 18 to 50 years with body weight of $>50 \mathrm{~kg}$ and body mass index of $18-32 \mathrm{~kg} / \mathrm{m}^{2}$. All subjects were medically healthy with clinically insignificant findings based on medical history, 12-lead electrocardiogram results and physical examination. Subjects with positive urine pregnancy test (female subjects only), previous exposure to pegfilgrastim or filgrastim or known allergy to PEG were excluded.

The safety population included all randomly assigned subjects who received $\geq 1$ dose of either study drug: pegfilgrastim-cbqv or pegfilgrastim. The safety population was used for all safety analyses in study CHS-1701-04, including ADAs, anti-G-CSF antibody titers for G-CSF-reactive ADA samples, and the coprimary NAb endpoint for all ADA-positive samples. In study CHS-170104, the ADA population included subjects who received both doses of the same study drug and who had $\geq 1$ ADA assessment after the second dose.

\section{Treatments}

On day 1 of each period, subjects were administered a single dose of pegfilgrastim-cbqv $(6 \mathrm{mg} / 0.6 \mathrm{ml}$ via sterile single-use prefilled syringe) or pegfilgrastim $(6 \mathrm{mg} / 0.6 \mathrm{ml}$ via sterile single-use prefilled syringe) subcutaneously per treatment assignment.

In studies CHS-1701-03 and CHS-1701-04, treatment was spaced $\geq 42$ days apart. In study CHS-1701-05, treatment of male subjects was spaced $\geq 28$ days apart; female subjects were dosed in 28-day intervals ( \pm 2 days) to decrease the intrasubject variability that could be introduced by dosing at different times throughout the subject's menstrual cycle.

\section{Assessments}

In studies CHS-1701-04 and CHS-1701-03, plasma samples for ADA testing were collected on day 1 (predose) and on days 13, 27 and 41 postdose for periods 1 and 2 (Fig. 1). In study CHS-1701-05, plasma samples for ADA testing were collected on day 1 (predose), on day 11 postdose in each period and on day 28 following the last dose of the study drug in period 3 (Fig. 1).

The testing strategy to assess immunogenicity followed a tiered approach (Fig. 2). All samples were tested in a screening assay to detect 


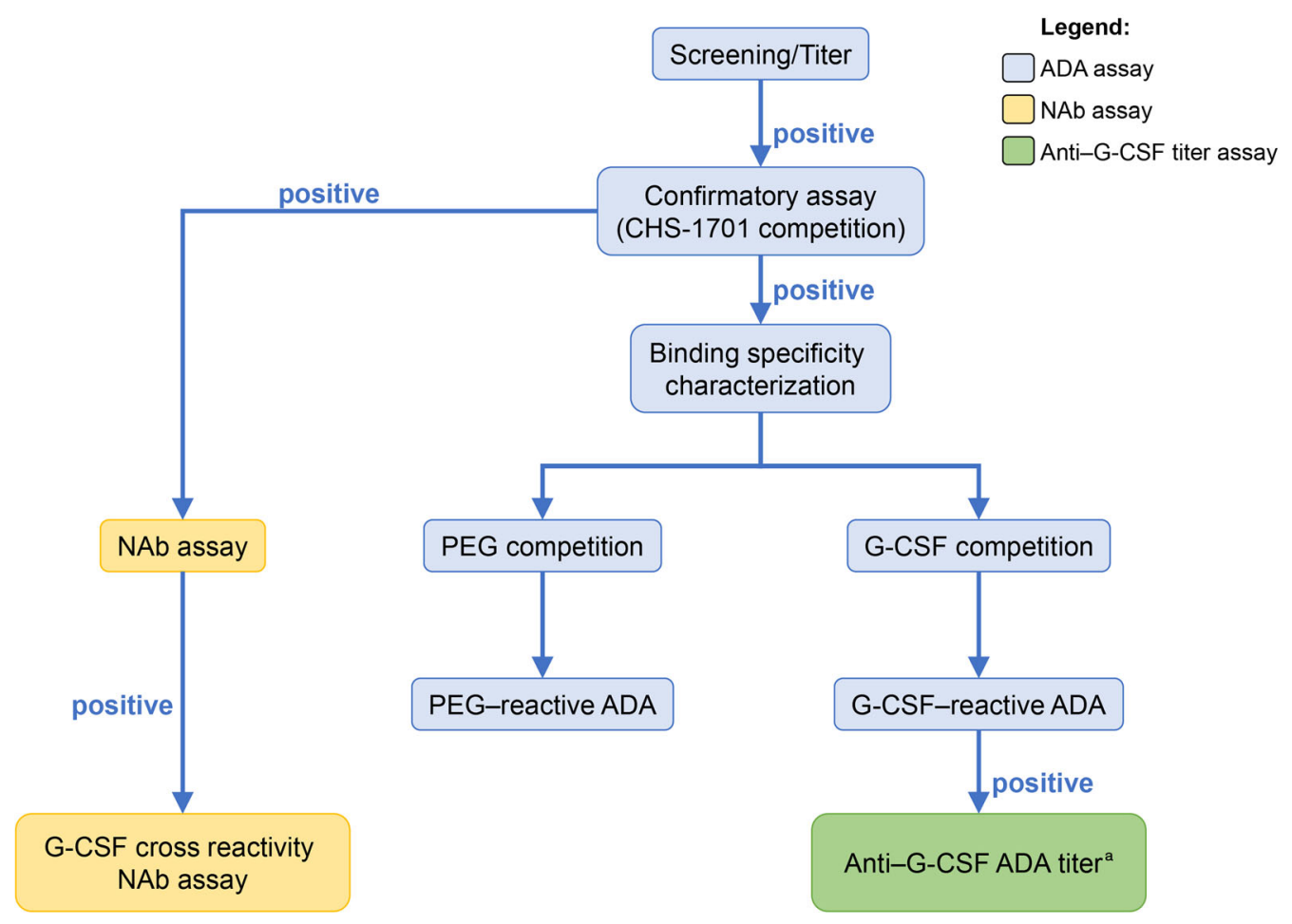

Fig. 2 Schematic of tiered immunogenicity assay approach. applies to study CHS-1701-04 only. $A D A$ antidrug antibody; $G$-CSF granulocyte-colony stimulating factor; $N A b$ neutralizing antibody; $P E G$ polyethylene glycol

ADAs. All ADA-positive samples were subsequently evaluated by titration to measure ADA titer and were tested in a confirmatory assay using competition with pegfilgrastim-cbqv. To evaluate the binding specificity of ADAs, confirmed positive samples were tested further for ADA binding to the PEG or filgrastim (GCSF) parts of the pegfilgrastim molecule using PEG and G-CSF competition assays. For study CHS-1701-04, samples that tested positive in the G-CSF competition assay (G-CSF-specific ADA) were further analyzed using a validated anti-G-CSF titer assay to determine the titer of G-CSF-specific ADA. All confirmed ADA-positive samples were tested in a validated cellbased NAb assay to determine whether the ADAs were neutralizing. NAb-positive samples were further tested in a cross-reactivity assay to determine neutralizing activity against human G-CSF.

The impact of ADAs on the PK of pegfilgrastim-cbqv or pegfilgrastim was evaluated by measuring plasma pegfilgrastim levels using a validated enzyme-linked immunosorbent assay
[11]. The impact of ADAs on the PD of pegfilgrastim-cbqv or pegfilgrastim was evaluated by measuring ANC in the blood, an established surrogate PD biomarker of clinical efficacy [11]. For safety analyses, treatment-emergent AEs (TEAEs), vital signs, laboratory variables and local injection site reactions (ISRs) were recorded. The impact of ADAs on TEAEs and ISRs was assessed.

\section{Anti-drug Antibody Assay}

In the screening assay, the presence of antipegfilgrastim antibodies was assessed with a validated electrochemiluminescence (ECL)bridging ADA assay method, using a mixture of biotinylated pegfilgrastim-cbqv and ruthenylated (SULFO-TAG ${ }^{\mathrm{TM}}$, Meso Scale Diagnostics, Rockville, MD, USA) pegfilgrastim-cbqv. Antipegfilgrastim-cbqv or anti-pegfilgrastim antibodies, if present in the samples, bivalently bound to the labeled pegfilgrastim-cbqv molecules and formed a bridge. This complex was 
then detected by excitation of the SULFO$\mathrm{TAG}^{\mathrm{TM}}$ via an electrochemical reaction of $\mathrm{Ru}$ (bpy) 3 to generate an ECL signal, which was read using the MSD Sector Imager 6000 (Meso Scale Diagnostics). Titer was determined by serial two-fold dilutions. Sample titer was defined as the highest dilution that tested positive in the titer assay multiplied by the minimal required dilution (MRD $=2$; thus, a titer of 2 was the lowest measurable titer). In the confirmatory assay, screen-positive samples were tested in the presence of excess pegfilgrastimcbqv to determine whether the signal was specific. All confirmed ADA-positive samples were further tested in the presence of excess PEG or G-CSF to characterize the binding specificity of the ADA to the PEG or filgrastim moieties of pegfilgrastim-cbqv.

\section{Anti-G-CSF Antibody Titer Assay}

Plasma samples from study CHS-1701-04 confirmed to have G-CSF-reactive ADAs were analyzed for titer of the anti-G-CSF antibodies using an ECL bridging assay. The titer assay used an MSD GOLD Streptavidin plate (Meso Scale Diagnostics) and a mixture of biotinylated and ruthenylated (SULFO-TAG ${ }^{\mathrm{TM}}$ ) G-CSF prepared using a recombinant glycosylated form of G-CSF expressed in HEK cells, which represents endogenous G-CSF. Rabbit polyclonal antiG-CSF antibody (Abcam \#9691) was used as a positive control. Test samples and controls were diluted 1:2 in assay buffer, and subsequent twofold serial dilutions of the neat test samples were prepared in matrix diluent (PNHP) followed by dilution to the MRD in assay buffer. After incubation of samples and subsequent washing, read buffer was added and the plate was read on the Sector Imager 6000 (Meso Scale Diagnostics). The titer was determined to be the highest-fold dilution that had a signal-to-noise $(\mathrm{S} / \mathrm{N})$ ratio greater than or equal to the statistically derived titer cutpoint $(\mathrm{S} / \mathrm{N}=1.28)$ multiplied by the $\mathrm{MRD}=$ 2. ADA-confirmed positive samples that had an $\mathrm{S} / \mathrm{N}$ ratio less than the titer cutpoint at MRD were reported as titer $<2$. Minimum titer measured in the anti-G-CSF antibody titer assay is 2 , which corresponds to $\sim 16.3 \mathrm{ng} / \mathrm{ml}$ concentration of anti-G-CSF rabbit polyclonal antibody-positive control. Also, $200 \mathrm{ng} / \mathrm{ml}$ of positive control generates a titer of 16-32; in comparison, $200 \mathrm{ng} /$ $\mathrm{ml}$ of the same positive control generates a titer of 4-8 in the ADA assays.

\section{NAb Assay}

The NAb assay utilized a cell line (NSF-60) that depends on G-CSF or mouse interleukin 3 (mIL-3) for proliferation. Cell proliferation after incubation with pegfilgrastim-cbqv was detected by a luminescent method (CellTiterGlo; Promega, Madison, WI, USA) to measure adenosine triphosphate (ATP), which is produced by metabolically active cells. The assay sensitivity was $137 \mathrm{ng} / \mathrm{ml}$. The plasma samples that were identified positive in the presence of pegfilgrastim-cbqv-induced cell proliferation were further tested in a NAb specificity assay, in the presence of mIL-3 instead of pegfilgrastim-cbqv. This was done to confirm that the signal observed in the screening assay was not due to matrix factors that nonspecifically inhibit cellular proliferation. Finally, a confirmatory assay, involving a Protein A/G/L Sepharose immunodepletion step, confirmed that the inhibition of the pegfilgrastim-cbqv signal was due to immunoglobulins $G$ or $M$ (IgG or IgM). All samples that were confirmed positive were further tested in the presence of human G-CSF to determine whether endogenous G-CSF was neutralized.

\section{Statistical Considerations}

The presence of ADAs and NAbs by treatment groups is presented in summary statistics. Incidence of ADAs, titers, binding specificity and time courses were assessed and summarized by treatment group. The impact of ADA on PK, PD and safety was also assessed. 


\section{RESULTS}

\section{CHS-1701-04}

\section{Subjects}

Study CHS-1701-04 (parallel-arm with repeat administration) was the key confirmatory study for the immunogenicity assessment of pegfilgrastim-cbqv and included 303 subjects; 151 subjects received pegfilgrastim-cbqv and 152 subjects received pegfilgrastim. The safety population included all 303 subjects, 242 of whom fulfilled the definition to be included in the ADA population. The major difference in the number of subjects between the two populations was due to a protocol deviation in which subjects received one dose of each treatment instead of two doses of the same treatment. Baseline characteristics are presented in Supplementary Table S1.

\section{Immunogenicity}

In the ADA population, the incidence of treatment-emergent, persistent ADAs with measurable titer (titer $\geq 2$ ) was $9.8 \%$ and $5.0 \%$ in subjects receiving pegfilgrastim-cbqv and pegfilgrastim, respectively (Table 1 ). In the safety population, the incidence of treatmentemergent ADAs was 32.2\% (39/121) in subjects receiving pegfilgrastim-cbqv and 23.9\% (28/ $117)$ in subjects receiving pegfilgrastim (Table 1). Most ADAs were transient (only present in period 1) or with low or unmeasurable titer. The incidence of preexisting ADA was 9.0\% (12/134) and 9.7\% (13/134) of subjects in the pegfilgrastim-cbqv and pegfilgrastim treatment groups, respectively (Table 1 ). In subjects with preexisting ADA, the incidence of boosted ADA response after treatment (defined as $\geq$ fourfold titer increase at any time after the first dose) was the same in the two treatment groups: three subjects $(2.3 \%)$ in the pegfilgrastim-cbqv group and three subjects $(2.3 \%)$ in the pegfilgrastim group.

ADA-binding reactivity was evaluated in the competition assays with an excess of PEG or G-CSF. The ADA binding reactivity was similar after treatment with pegfilgrastim-cbqv or pegfilgrastim. In both treatment groups, most ADAs were PEG-reactive (i.e., reacted with PEG only or with PEG and G-CSF): in the safety population, $26.4 \%$ of subjects in the pegfilgrastim-cbqv group compared with $20.5 \%$ in the pegfilgrastim group (Table 1). A similar trend was observed for the subjects with a persistent ADA titer $\geq 2$ : 11/12 ADApositive subjects in the pegfilgrastim-cbqv group and all six subjects in the pegfilgrastim group had PEG-reactive ADAs (either PEG only or PEG and G-CSF). For one subject in the pegfilgrastim-cbqv group, binding specificity could not be determined (Table 1). Therefore, the small numerical difference between pegfilgrastim-cbqv and pegfilgrastim was due primarily to anti-PEG antibodies. In both treatment groups, ADA-positive subjects with ADA-binding specificity to PEG and G-CSF had relatively low levels of inhibition in the G-CSF competition assay and much stronger inhibition in the PEG competition assay, indicating that the signal was primarily driven by PEGreactive ADAs. No subject had ADAs that reacted only with G-CSF. All samples with PEG- or G-CSF-reactive ADAs were further characterized by measuring anti-G-CSF antibody titers and the titer kinetics. Three subjects had measurable anti-G-CSF titers (two in pegfilgrastim-cbqv and one in pegfilgrastim). The anti-G-CSF antibody titers were transient and were measured at the earliest time point after the first dose (period 1); they then decreased and were only detected in period 1 (Supplementary Table S2). Overall, the low incidence and transient profile of anti-G-CSF titers indicate no significant difference between pegfilgrastim-cbqv and pegfilgrastim.

The time course of ADA response, represented as percentage of ADA-positive subjects (ADA incidence) at each time point, was similar after treatment with pegfilgrastim-cbqv or pegfilgrastim. In both treatment groups, the highest ADA incidence was observed at the first tested time point (study day 13). Thus, ADAs developed rapidly after the first dose and decreased thereafter, with few subjects becoming ADA positive after the second dose (Table 4). Similarly, the ADA titers did not increase after the second dose and were similar in the two treatment groups. The highest titers of 
Table 1 Incidence of subjects with ADAs in CHS-1701-04

\begin{tabular}{|c|c|c|}
\hline & $\begin{array}{l}\text { Pegfil-cbqv } \\
(N=134)\end{array}$ & $\begin{array}{l}\text { Pegfil } \\
(N=134)\end{array}$ \\
\hline Preexisting (baseline) ADAs, $n(\%)$ & $12(9.0)$ & $13(9.7)$ \\
\hline \multicolumn{3}{|l|}{ Binding specificity, $n$ (\%) } \\
\hline PEG & $12(9.0)$ & $10(7.5)$ \\
\hline PEG/G-CSF & $2(1.5)$ & 0 \\
\hline PEG Only & $10(7.5)$ & $10(7.5)$ \\
\hline G-CSF Only & 0 & 0 \\
\hline None $^{\mathrm{a}}$ & 0 & $3(1.1)$ \\
\hline Treatment-emergent ADAs (safety population), $n$ (\%) & $39 / 121(32.2)$ & $28 / 117(23.9)$ \\
\hline \multicolumn{3}{|l|}{ Binding specificity, $n(\%)$} \\
\hline PEG & $32 / 121(26.4)$ & $24 / 117(20.5)$ \\
\hline PEG/G-CSF & $9 / 121(7.4)$ & $7 / 117(6.0)$ \\
\hline PEG only & $23 / 121(19.0)$ & $17 / 117(14.5)$ \\
\hline G-CSF only & 0 & 0 \\
\hline None $^{\mathrm{a}}$ & $7 / 121(5.8)$ & $4 / 117(3.4)$ \\
\hline Treatment-emergent, titer $\geq 2$ and persistent $\mathrm{ADA}$ (ADA population), $n$ (\%) & $12 / 122(9.8)$ & $6 / 120(5.0)$ \\
\hline \multicolumn{3}{|l|}{ Binding specificity, $n(\%)$} \\
\hline PEG & $11 / 122(9.0)$ & $6 / 120(5.0)$ \\
\hline PEG/G-CSF & $6 / 122(4.9)$ & $4 / 120(3.3)$ \\
\hline PEG only & $5 / 122(4.1)$ & $2 / 120(1.7)$ \\
\hline G-CSF only & 0 & 0 \\
\hline None & $1 / 122(0.8)$ & 0 \\
\hline
\end{tabular}

Data from site 004 were excluded because the wrong study drug was administered in period 2

$A D A$ antidrug antibody, G-CSF granulocyte colony-stimulating factor, $P E G$ polyethylene glycol, Pegfil pegfilgrastim, Pegfil-cbqv pegfilgrastim-cbqv

${ }^{a}$ Not binding to PEG or G-CSF

${ }^{\mathrm{b}}$ Negative result or no result at baseline (period 1 day 1 predose) and positive result after period 1 dose and prior to period 2 dose. $\%=100^{*} n / N$, in which $N$ was the number of subjects with ADA-assessed period 1 postdose. Subjects positive at baseline were not included in $N^{N}$

${ }^{\mathrm{c}}$ Negative result at all visits prior to period 2 dose and positive result in period 2 postdose. $\%=n / N$, in which $N$ was the total number of subjects with ADA assessed period 2 postdose, regardless of whether the result was positive or negative. Subjects positive at any visits prior to period 2 dose were not included in $N^{p}$

treatment-emergent ADAs were observed in period 1 after the first dose, with a maximum titer of 64 in the pegfilgrastim-cbqv group and 256 in the pegfilgrastim group.
NAbs were not detected after dosing in either treatment group. However, preexisting PEG-reactive ADAs with pegfilgrastim-neutralizing activity were detected in two subjects at 
the predose time point only. Of note, the NAbs were not able to neutralize the activity of human G-CSF, suggesting that these anti-PEG NAbs may inhibit the activity of pegfilgrastim by steric hindrance. No clinical impact of the preexisting NAbs was identified in the conducted PK, PD and safety analyses.

\section{Impact of ADAs on PK and PD}

The impact of ADAs was evaluated by comparing key PK and PD parameters between ADAnegative and -positive subjects. For the PK analysis, maximum plasma concentration $\left(C_{\max }\right)$ and area under the plasma concentration time curve calculated from time 0 to last measurable concentration $\left(\mathrm{AUC}_{0 \text {-last }}\right)$ were evaluated. For the PD analysis, maximum ANC $\left(\mathrm{ANC}_{\max }\right)$ and area under the ANC time curve from time 0 to last measurable observation $\left(\mathrm{ANC} \mathrm{AUC}_{0 \text {-last }}\right.$ ) were evaluated. This analysis demonstrated no impact of ADA on PK or PD in either study period. $\mathrm{PK}\left(C_{\max }\right.$ and $\left.\mathrm{AUC}_{0 \text {-last }}\right)$ and $\mathrm{PD}\left(\mathrm{ANC}_{\max }\right.$ and $\left.\mathrm{ANC} \mathrm{AUC}_{0 \text {-last }}\right)$ parameters in ADA-positive subjects were within the range observed in ADA-negative subjects in each study period in both treatment groups (Figs. 3 and 4).

\section{Impact of ADAs on Safety}

The safety profiles for pegfilgrastim-cbqv and pegfilgrastim in this study were consistent with the known pegfilgrastim safety profile and appeared to be unaffected by the presence of $\mathrm{ADA}$. The overall incidence of any $\mathrm{AE}$ was $89.8 \%$ for ADA-negative subjects and $91.0 \%$ for subjects with treatment-emergent ADA (Table 2). Furthermore, the incidence of the most common AEs (e.g., various manifestations of musculoskeletal pain, headache) was similar in ADA-negative and ADA-positive subjects. The overall incidence of any AE was $89.7 \%$ in the pegfilgrastim-cbqv group and $92.9 \%$ in the pegfilgrastim group for subjects with treatmentemergent ADA (Table 2). The incidence of ISRs by ADA status was also evaluated by period. The incidence of ISRs was comparable in ADAnegative and ADA-positive subjects (Table 3).

\section{Pooled Analyses}

\section{Subjects}

The immunogenicity similarity between pegfilgrastim-cbqv and pegfilgrastim was further assessed in a pooled analysis that included data from CHS-1701-04, CHS-1701-05 and CHS1701-03. The safety population for this pooled analysis included 487 subjects with ADA assessed in period 1 postdose before dosing of period 2. Subjects positive at baseline (period 1 day 1) were not included. The CHS-170104, CHS-1701-05 and CHS-1701-03 studies included 268, 117 and 102 subjects, respectively. Baseline characteristics were similar between the two treatment groups in study CHS-1701-04 (Supplementary Table S1) and in the pooled analysis (Supplementary Table S3). Only results from period 1 were evaluated in this pooled analysis because the second dose in the crossover studies, CHS-1701-03 and CHS1701-05, would have confounded the analysis. The use of only period 1 is relevant, as most immune responses occurred in period 1 in all studies, with few new treatment-emergent ADAs identified after the second and no new treatment-emergent ADAs identified after the third dose (Table 4).

\section{Immunogenicity}

In the pooled analysis, treatment-emergent ADAs in period 1 were detected in $31.6 \%$ (72/ 228 ) of subjects receiving pegfilgrastim-cbqv and in $29.3 \%(76 / 259)$ of subjects receiving pegfilgrastim (Table 5). The incidence of treatment-emergent ADAs after the first dose of pegfilgrastim-cbqv and pegfilgrastim was comparable between studies and similar in the two treatment groups: $33.1 \%$ versus $25.0 \%$ in CHS1701-04, 28.6\% versus $33.3 \%$ in CHS-1701-05 and $30.0 \%$ versus $34.6 \%$ in CHS-1701-03 (Table 5).

Binding-specificity assays showed that in both treatment groups the treatment-emergent ADAs were binding PEG (PEG only or PEG and G-CSF) in most subjects (Table 5). Treatmentemergent ADAs reactive with only G-CSF were not detected in any of the three studies (Table 5). In addition, the time course and titers 
(A) $\mathrm{C}_{\max }$ in Study CHS-1701-04

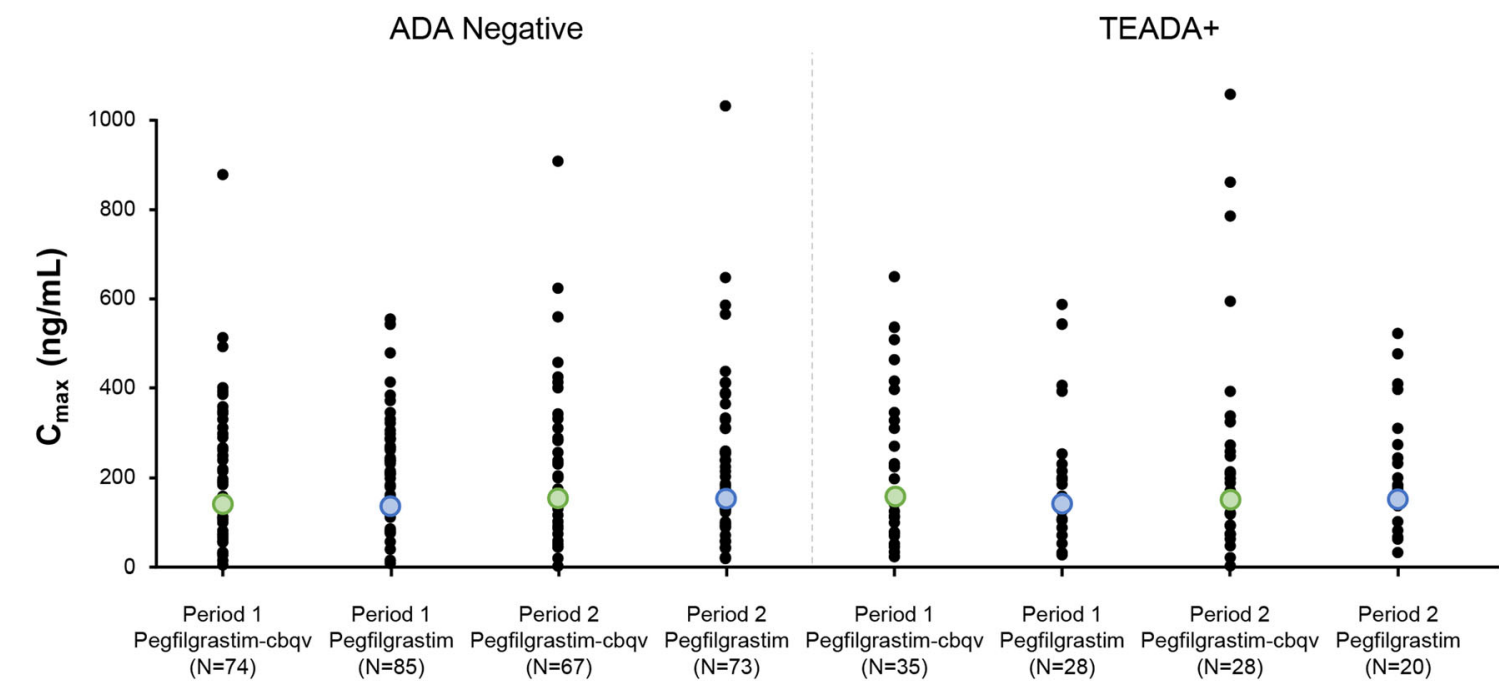

(B) AUC $_{0 \text {-last }}$ in Study CHS-1701-04

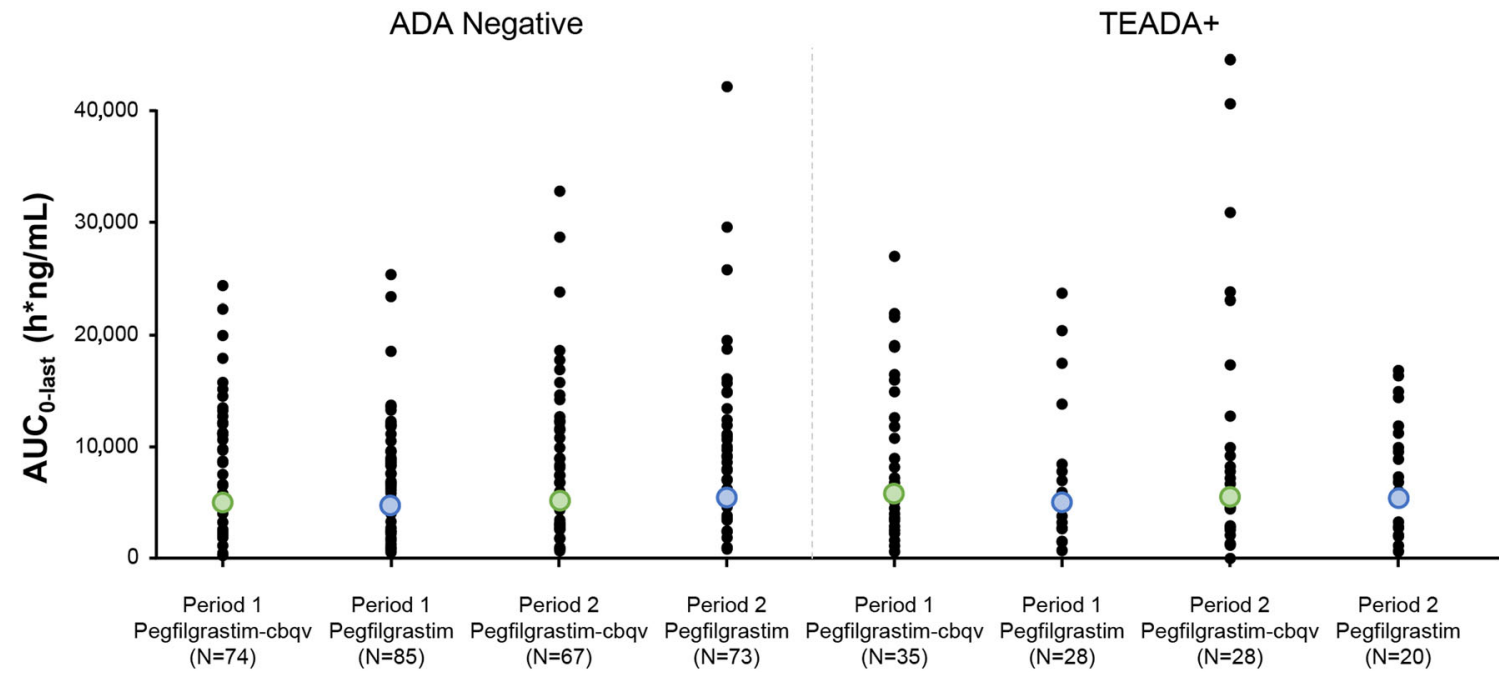

Fig. 3 Comparison of the PK parameters a $C_{\max }$ and b $\mathrm{AUC}_{0 \text {-last }}$ between $\mathrm{ADA}$-negative subjects and subjects with treatment-emergent ADA across all periods in study CHS-1701-04. The geometric means are represented by colored circles. Figure includes subjects with sufficient

of the ADA response was similar in all studies. Treatment-emergent NAbs were not detected in any subject across the three studies. However, preexisting PEG-reactive ADAs with pegfilgrastim-neutralizing activity were detected in four subjects at the predose time point only. pharmacokinetics samples to calculate each parameter. $A D A$ antidrug antibody, $A U C_{\text {o-last }}$ area under the plasma concentration-time curve calculated from time 0 to last measurable concentration, $C_{\max }$ maximum plasma concentration, $P K$ pharmacokinetics, $T E$ treatment-emergent

\section{DISCUSSION}

Biologic therapeutics can trigger immune responses in patients. The resulting ADAs may have no clinical impact or they may affect the bioavailability, efficacy and safety of the therapeutic to various degrees [12]. Therefore, the 
(A) ANC $_{\text {max }}$ in Study CHS-1701-04

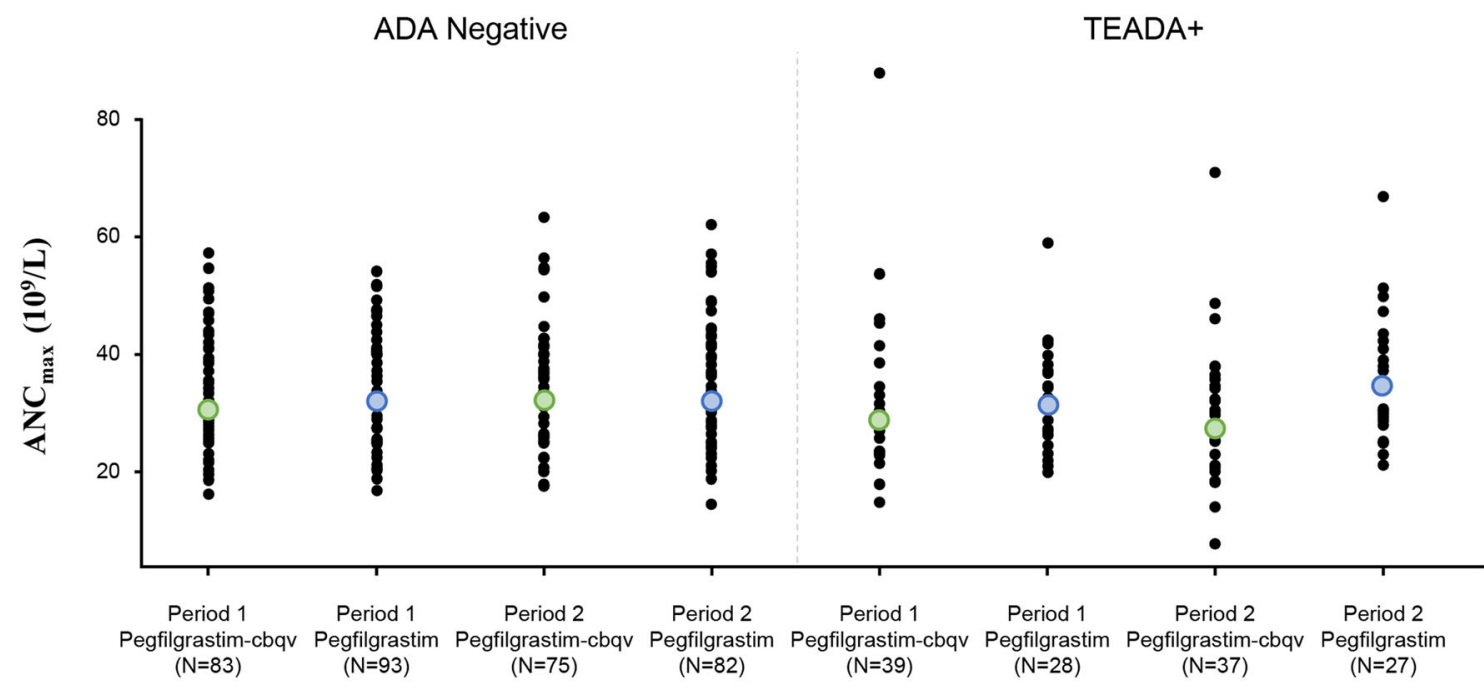

(B) ANC AUC -last $_{\text {in Study CHS-1701-04 }}$

ADA Negative

TEADA+

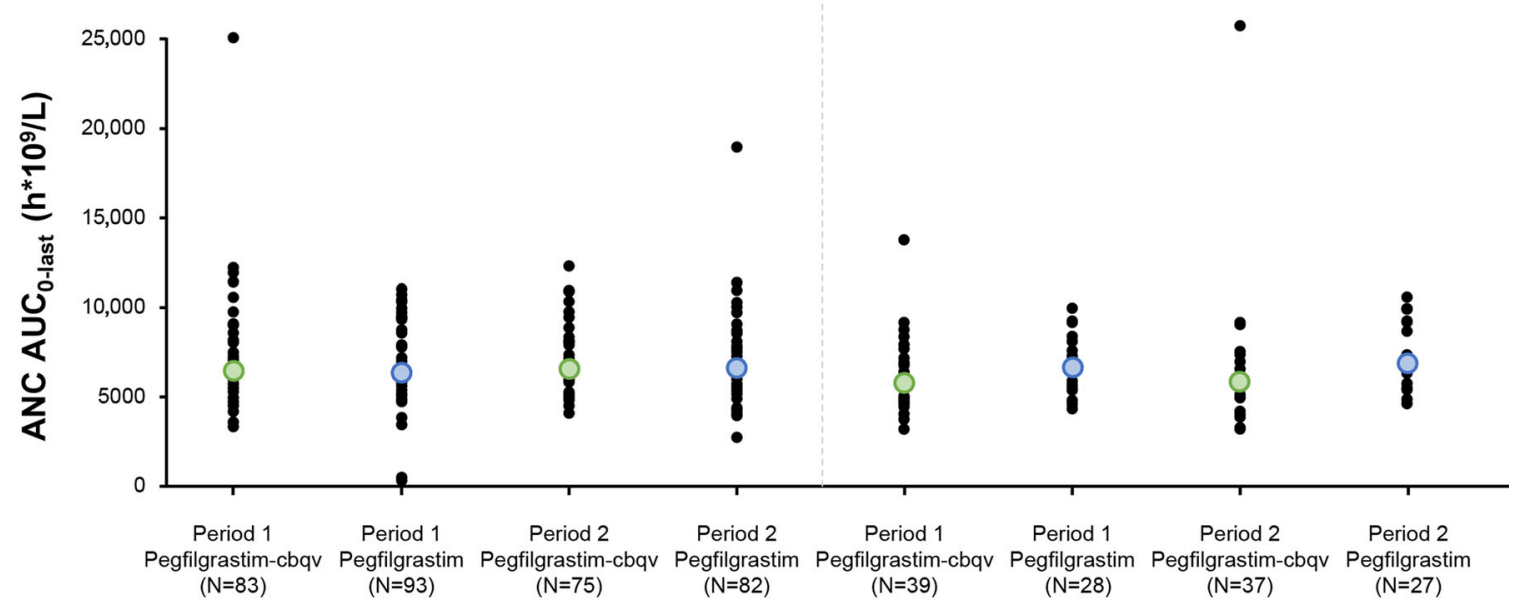

Fig. 4 Comparison of the PD parameters a $\mathrm{ANC}_{\max }$ and

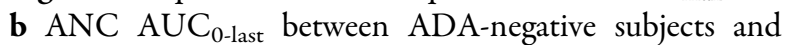
subjects with treatment-emergent ADA across all periods in study CHS-1701-04. The geometric means are represented by colored circles. $A D A$ antidrug antibody,

$A U C_{0 \text {-last }}$ area under the plasma concentration-time curve calculated from time 0 to last measurable concentration, $A N C_{\max }$ maximum observed absolute neutrophil count, $T E$ treatment emergent

immunogenicity profile is a critical quality attribute of biologic therapeutics and their biosimilars [13]. Pegfilgrastim-cbqv (UDE$\mathrm{NYCA}^{\circledR}$ ) is a biosimilar of pegfilgrastim $\left(\right.$ Neulasta ${ }^{\circledR}$ ), a pegylated, long-acting form of G-CSF, indicated for the prophylaxis of febrile neutropenia in patients with cancer who receive myelosuppressive chemotherapy $[7,10]$. The PK

and PD bioequivalence of pegfilgrastim-cbqv has been previously reported [11], and pegfilgrastimcbqv has been approved by the FDA and EMA for the same indications as its reference product, pegfilgrastim $[7,10]$. Consistent with regulatory guidance $[1,4]$, the immunogenicities of pegfilgrastim-cbqv and pegfilgrastim were compared in the clinical development program. 
Table 2 Incidence of adverse events occurring in $>5 \%$ of subjects by ADA status (CHS-1701-04)

\begin{tabular}{lcl}
\hline & $\begin{array}{l}\text { ADA-negative } \\
(\boldsymbol{N}=\mathbf{1 7 6})\end{array}$ & $\begin{array}{l}\text { Treatment-emergent ADA } \\
(\boldsymbol{N}=\mathbf{6 7})\end{array}$ \\
\hline Subjects with any AE & $158(89.8)$ & $61(91.0)$ \\
Back pain & $121(68.8)$ & $50(74.6)$ \\
Headache & $108(61.4)$ & $41(61.2)$ \\
Pain in extremity & $43(24.4)$ & $11(16.4)$ \\
Pain (unspecified) & $31(17.6)$ & $12(17.9)$ \\
Arthralgia & $33(18.8)$ & $9(13.4)$ \\
Nausea & $20(11.4)$ & $7(10.4)$ \\
Neck pain & $16(9.1)$ & $6(9.0)$ \\
Musculoskeletal chest pain & $13(7.4)$ & $2(3.0)$ \\
Vomiting & $12(6.8)$ & $3(4.5)$ \\
Dizziness & $7(4.0)$ & $5(7.5)$ \\
Musculoskeletal pain & $7(4.0)$ & $4(6.0)$ \\
Muscle spasms & $5(2.8)$ & $4(6.0)$ \\
\hline
\end{tabular}

Percentage was calculated using the number of subjects in the column header as the denominator. AEs were collected from the time of dosing on day 1 of period 1 through day 41 of period 2. Therefore, all AEs were considered treatment emergent. All AEs were coded using MedDRA version 18.0. Data from site 004 were excluded because the wrong study drug was administered in period 2

$A D A$ antidrug antibody, $A E$ adverse event

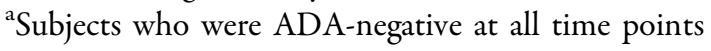

Table 3 Injection site reactions by ADA status (CHS-1701-04, safety population)

\begin{tabular}{|c|c|c|c|c|}
\hline \multirow[t]{2}{*}{ ISR } & \multicolumn{2}{|c|}{ ADA-negative ${ }^{a}$} & \multicolumn{2}{|c|}{ Treatment-emergent ADA } \\
\hline & $\begin{array}{l}\text { Period } 1 \\
N=176\end{array}$ & $\begin{array}{l}\text { Period } 2 \\
N=157\end{array}$ & $\begin{array}{l}\text { Period } 1 \\
N=67\end{array}$ & $\begin{array}{l}\text { Period 2 } \\
N=64\end{array}$ \\
\hline \multicolumn{5}{|l|}{ Any, $n(\%)^{b}$} \\
\hline Mild & $32(18.2)$ & $27(17.2)$ & $9(13.4)$ & $11(17.2)$ \\
\hline Moderate & 0 & $2(1.3)$ & 0 & $1(1.6)$ \\
\hline Severe & $1(0.6)$ & 0 & 0 & 0 \\
\hline
\end{tabular}

Data from site 004 were excluded because the wrong study drug was administered in period 2

$A D A$ antidrug antibody, ISR injection site reaction

${ }^{\mathrm{a}} \mathrm{Subjects}$ who were ADA negative at all time points

${ }^{\mathrm{b}}$ Summarizes the worst postdose assessment within each period per subject 
Table 4 Treatment-emergent ADA by period and treatment (safety population)

\begin{tabular}{|c|c|c|c|c|c|c|c|}
\hline \multirow[t]{2}{*}{ Subjects with } & \multicolumn{2}{|c|}{ Study CHS-1701-04 } & \multicolumn{2}{|c|}{ Study CHS-1701-03 } & \multicolumn{3}{|c|}{ Study CHS-1701-05 } \\
\hline & $\begin{array}{l}\text { Pegfil- } \\
\text { cbqv/ } \\
\text { Pegfil- } \\
\text { cbqv } \\
(N=134)\end{array}$ & $\begin{array}{l}\text { Pegfil/ } \\
\text { Pegfil } \\
(N=134)\end{array}$ & $\begin{array}{l}\text { Pegfil- } \\
\text { cbqv/ } \\
\text { Pegfil } \\
(N=58)\end{array}$ & $\begin{array}{l}\text { Pegfil/ } \\
\text { Pegfil- } \\
\text { cbqv } \\
(N=111)\end{array}$ & $\begin{array}{l}\text { Pegfil- } \\
\text { cbqv/ } \\
\text { Pegfil/ } \\
\text { Pegfil } \\
(N=43)\end{array}$ & $\begin{array}{l}\text { Pegfil/ } \\
\text { Pegfil- } \\
\text { cbqv/ } \\
\text { Pegfil } \\
(N=37)\end{array}$ & $\begin{array}{l}\text { Pegfil/ } \\
\text { Pegfil/ } \\
\text { Pegfil- } \\
\text { cbqv } \\
(N=42)\end{array}$ \\
\hline $\begin{array}{l}\text { Treatment-emergent ADA in } \\
\text { period } 1,{ }^{\mathrm{a}} n / N^{\prime}(\%)\end{array}$ & $\begin{array}{r}38 / 121 \\
(31.4)\end{array}$ & $\begin{array}{r}28 / 117 \\
(23.9)\end{array}$ & $\begin{array}{l}15 / 50 \\
(30.0)\end{array}$ & $\begin{array}{l}18 / 52 \\
(34.6)\end{array}$ & $\begin{array}{l}12 / 42 \\
(28.6)\end{array}$ & $\begin{array}{l}12 / 36 \\
(33.3)\end{array}$ & $\begin{array}{l}13 / 39 \\
(33.3)\end{array}$ \\
\hline $\begin{array}{l}\text { Newly developed treatment- } \\
\text { emergent ADA in } \\
\text { period 2, }{ }^{\mathrm{b}} n / N^{\prime}(\%)\end{array}$ & $1 / 76(1.3)$ & $0 / 81(0)$ & $\begin{array}{l}1 / 30 \\
(3.3)\end{array}$ & $0 / 31(0.0)$ & $1 / 22(4.5)$ & $0 / 17(0.0)$ & $1 / 22(4.5)$ \\
\hline $\begin{array}{l}\text { Newly developed treatment- } \\
\text { emergent ADA in } \\
\text { period } 3,^{c} n / N^{\prime}(\%)\end{array}$ & N/A & N/A & N/A & N/A & $0 / 13(0.0)$ & $0 / 15(0.0)$ & $0 / 17(0.0)$ \\
\hline
\end{tabular}

$A D A$, antidrug antibody, Pegfil pegfilgrastim, Pegfil-cbqv pegfilgrastim-cbqv

${ }^{a}$ Negative result or no result at baseline (period 1 day 1 predose) and positive result after period 1 dose and prior to period 2 dose. $\%=100^{*} n / N^{\prime}$, in which $N^{\gamma}$ was the number of subjects with ADA assessed postdose in the respective periods. Subjects positive at baseline were not included in $N$

${ }^{b}$ Negative result at all visits prior to period 2 dose and positive result in period 2 postdose. $\%=n / N$, where $N$ was the total number of subjects with ADA assessed period 2 postdose, regardless of whether the result was positive or negative. Subjects positive at any visits prior to period 2 dose were not included in $N^{p}$

${ }^{\circ}$ Negative result at all visits in period 1 , period 2 and period 3 day 1 predose and positive result period 3 postdose. $\%=n / N$, where $N^{\prime}$ is the total number of subjects with ADA assessed period 3 postdose, regardless of whether the result was positive or negative. Subjects positive at any visits in period 1 (including period 1 day 1), period 2 and period 3 day 1 predose were not included in $N$

Pegfilgrastim treatment is associated with a low incidence of treatment-emergent ADAs and an absence of clinically impactful immunogenicity $[7,14,15]$. However, there is the theoretical potential for ADAs binding to the G-CSF portion of pegfilgrastim to also bind to and neutralize endogenous G-CSF, which might have a clinical impact. In addition, PEG-binding antibodies have been found in treatment-naive individuals, presumably due to prior exposure to PEG and PEG derivatives [16, 17]. This report presents a thorough immunogenicity assessment from study CHS-1701-04, which was specifically designed to assess immunogenicity, and from a pooled analysis that included two additional studies conducted to confirm the bioequivalence of pegfilgrastim-cbqv and pegfilgrastim (CHS-1701-03 and CHS-1701-05).
Study CHS-1701-04 was a randomized, doubleblind, two-period parallel-arm study whose primary objective was to assess the immunogenicity of pegfilgrastim-cbqv versus pegfilgrastim in healthy subjects. The impacts of ADAs and NAbs on the PK, PD and safety profile of pegfilgrastim-cbqv were secondary objectives.

In the CHS-1701-04 study, a slightly higher incidence of ADAs was detected in the pegfilgrastim-cbqv group. However, when data from all clinical studies were pooled, the incidence of ADAs was similar after treatment with pegfilgrastim-cbqv or pegfilgrastim. Furthermore, the time courses of the immune response and the titers were similar. In both treatment groups, the ADAs were generated early after the first dose and subsequently decreased 
Table 5 Treatment-emergent ADA in period 1 of studies CHS-1701-03, CHS-1701-04, CHS-1701-05 and pooled (safety population)

\begin{tabular}{|c|c|c|c|c|c|c|c|c|}
\hline & \multicolumn{2}{|c|}{$\begin{array}{l}\text { Study CHS-1701-04 } \\
\text { (including site 004) }\end{array}$} & \multicolumn{2}{|c|}{$\begin{array}{l}\text { Study CHS- } \\
1701-05\end{array}$} & \multicolumn{2}{|c|}{$\begin{array}{l}\text { Study CHS- } \\
1701-03\end{array}$} & \multicolumn{2}{|c|}{ All Studies Pooled } \\
\hline & $\begin{array}{l}\text { Pegfil- } \\
\text { cbqv } \\
\left(n^{\prime}=136\right)\end{array}$ & $\begin{array}{l}\text { Pegfil } \\
\left(n^{\prime}=132\right)\end{array}$ & $\begin{array}{l}\text { Pegfil- } \\
\text { cbqv } \\
\left(n^{\prime}=42\right)\end{array}$ & $\begin{array}{l}\text { Pegfil } \\
\left(n^{\prime}=75\right)\end{array}$ & $\begin{array}{l}\text { Pegfil- } \\
\text { cbqv } \\
\left(n^{\prime}=50\right)\end{array}$ & $\begin{array}{l}\text { Pegfil } \\
\left(n^{\prime}=52\right)\end{array}$ & $\begin{array}{l}\text { Pegfil- } \\
\text { cbqv } \\
\left(n^{\prime}=228\right)\end{array}$ & $\begin{array}{l}\text { Pegfil } \\
\left(n^{\prime}=259\right)\end{array}$ \\
\hline $\begin{array}{l}\text { Treatment-emergent } \\
\text { ADA, } n(\%) \text { (period } \\
1 \text { only) }\end{array}$ & $45(33.1)$ & $33(25.0)$ & $12(28.6)$ & $25(33.3)$ & $15(30.0)$ & $18(34.6)$ & $72(31.6)$ & $76(29.3)$ \\
\hline \multicolumn{9}{|l|}{ Binding specificity } \\
\hline PEG only & $28(20.6)$ & $19(14.4)$ & $6(14.3)$ & $14(18.7)$ & $11(22.0)$ & $15(28.8)$ & $45(19.7)$ & $48(18.5)$ \\
\hline PEG and G-CSF & $8(5.9)$ & $7(5.3)$ & $1(2.4)$ & $1(1.3)$ & $3(6.0)$ & $1(1.9)$ & $12(5.3)$ & $9(3.5)$ \\
\hline G-CSF only & 0 & 0 & 0 & 0 & 0 & 0 & 0 & 0 \\
\hline None & $9(6.6)$ & $7(5.3)$ & $4(9.5)$ & $10(13.3)$ & 0 & $1(1.9)$ & $13(5.7)$ & $18(6.9)$ \\
\hline Not applicable & 0 & 0 & $1(2.4)$ & $0(0.0)$ & $1(2.0)$ & $1(1.9)$ & $2(0.9)$ & $1(0.4)$ \\
\hline
\end{tabular}

None, ADA not reactive with PEG or G-CSF. Not applicable: binding specificity was not characterized. $\%=n / n$; $n=$ negative result or no result at baseline and positive result at period 1 postdose. $n$ ' $=$ total number of subjects with ADA in a treatment arm assessed period 1 postdose before dosing of period 2. Subjects positive at baseline (period 1 day 1$)$ are not included in $n$,

$A D A$ antidrug antibody, G-CSF granulocyte colony-stimulating factor, PEG polyethylene glycol, Pegfil pegfilgrastim, Pegfilcbqv pegfilgrastim-cbqv

throughout the study. The ADA titers were generally low and similar in the two treatment groups and did not increase after the second dose. Importantly, binding-specificity assays showed that most ADAs were binding the PEG moiety of pegfilgrastim-cbqv, with no sample showing binding to only the G-CSF portion of pegfilgrastim. The samples with ADAs binding to both PEG and G-CSF were further evaluated in a validated anti-G-CSF titer assay. Overall, the low incidence and transient profile of antiG-CSF titers indicates no significant difference between pegfilgrastim-cbqv and pegfilgrastim. Furthermore, none of the treatment-emergent ADAs were neutralizing. Taken together, these findings show that the small differences in ADA incidence between the pegfilgrastim-cbqv and pegfilgrastim treatment groups were driven by non-neutralizing, low titer, PEG-reactive ADAs, which are commonly present in healthy subjects $[16,17]$.
To assess whether ADAs may have an impact on the PK and PD of pegfilgrastim-cbqv in study CHS-1701-04, drug plasma concentrations $\left(C_{\max }\right.$ and $\left.\mathrm{AUC}_{0 \text {-last }}\right)$ and the $\mathrm{PD}$ response were measured in all subjects. No significant differences were observed in $\mathrm{PK}\left(C_{\max }\right.$ and $\left.\mathrm{AUC}_{0 \text {-last }}\right)$ and PD analyses in ADA-positive and ADAnegative subjects. This finding is consistent with reports that PEG-reactive antibodies that have been found to affect efficacy, PK and/or safety of pegylated products [18] are present at much higher titers than those observed in this study. The safety profile of pegfilgrastim-cbqv was consistent with that previously reported for pegfilgrastim-cbqv [11] and pegfilgrastim [7], with no association to ADA status.

Overall, this comparison of PK, PD and safety in ADA-positive and -negative subjects confirmed that differences observed in ADA incidence were not clinically meaningful and were driven by non-neutralizing, low titer, PEG- 
reactive ADAs, which are commonly present in healthy subjects. These data further show that treatment-emergent anti-pegfilgrastim antibodies were not clinically meaningful, as reported in the literature $[7,14,16]$.

A limitation of this study was the use of nonidentical syringes containing each treatment. This was overcome by implementing measures to ensure that subjects and investigational staff were blinded to the subject treatment. The strengths of the presented studies and analyses include the large population size of the pooled analysis, which increased the robustness of the data, and the study design evaluating immunogenicity in healthy subjects, who are immunocompetent and have fewer confounding factors (e.g., comorbidities, concomitant medications). Healthy subjects therefore represent the most sensitive study population in which to evaluate potentially subtle differences in immunogenicity [4].

\section{CONCLUSION}

The presented data from three clinical studies with $>500$ subjects demonstrate that the immunogenicity of pegfilgrastim-cbqv and its reference product pegfilgrastim are highly similar and confirm the low immunogenic potential of both drugs. The evaluation of PK, PD and safety in ADA-positive and -negative subjects showed that treatment-emergent ADAs had no clinically meaningful impact. Immunogenicity data from the presented clinical studies support the overall demonstration of biosimilarity between pegfilgrastim-cbqv and pegfilgrastim.

\section{ACKNOWLEDGEMENTS}

We thank the participants of the study.

Funding. This study and medical writing support, along with the Rapid Service and Open Access Fees, were funded by Coherus BioSciences (Redwood City, CA).

Medical Writing and Editorial Assistance. Medical writing and editorial assistance was provided by Monique N. O'Leary, PhD, of ApotheCom (San Francisco, CA) and funded by Coherus BioSciences (Redwood City, CA).

Authorship. All named authors meet the International Committee of Medical Journal Editors (ICMJE) criteria for authorship for this manuscript, take responsibility for the integrity of the work as a whole, and have given their approval for this version to be published.

Author Contributions. All authors contributed to the study conception, design, and conduct. Material preparation, data collection, and analysis were performed by Francesca Civoli and Helen Tang. The first draft of the manuscript was written by Francesca Civoli and all authors commented on previous versions of the manuscript. All authors read and approved the final manuscript.

Disclosures. Francesca Civoli, Barbara Finck, Helen Tang, Jennifer Hodge, Hillary O'Kelly, and Vladimir Vexler are employees of and owned stock in Coherus BioSciences (Redwood City, CA). Jennifer Hodge is now an employee of Myovant Sciences Inc. (Brisbane, CA).

Compliance with Ethics Guidelines. The protocol and informed consent form were submitted to and approved by the sites' institutional review boards (IRBs) before initiation of the study. IRB approval was obtained from Alpha IRB (San Clemente, CA, USA), Chesapeake IRB (currently Advarra; Columbia, MD, USA), IntegReview Independent IRB (Austin, TX, USA), Midlands IRB (Overland Park, KS, USA), Schulman IRB (Cincinnati, OH, USA), and Western IRB (Puyallup, WA, USA). This study was conducted in accordance with all applicable laws and regulations, and it complied with the International Conference for Harmonisation E6 Guideline on Good Clinical Practice and the Declaration of Helsinki.

Consent to Participate. The rationale of the study, procedural details, and investigational goals were explained to each subject, along with potential risks and benefits. Each subject was assured of his/her right to withdraw from the 
study at any time. Before the initiation of any study procedures, each subject signed and dated an approved informed consent form.

Data Availability. Coherus BioSciences will review all requests for data and consider providing data on a case-by-case basis. As the studies reported in this publication support the biosimilarity assessment for an FDA- and EMAapproved biosimilar, pegfilgrastim-cbqv, the data have undergone extensive review by the FDA and EMA. Coherus BioSciences does not generally provide raw or deidentified datasets to investigators or journals.

Open Access. This article is licensed under a Creative Commons Attribution-NonCommercial 4.0 International License, which permits any non-commercial use, sharing, adaptation, distribution and reproduction in any medium or format, as long as you give appropriate credit to the original author(s) and the source, provide a link to the Creative Commons licence, and indicate if changes were made. The images or other third party material in this article are included in the article's Creative Commons licence, unless indicated otherwise in a credit line to the material. If material is not included in the article's Creative Commons licence and your intended use is not permitted by statutory regulation or exceeds the permitted use, you will need to obtain permission directly from the copyright holder. To view a copy of this licence, visit http://creativecommons.org/licenses/bync/4.0/.

\section{REFERENCES}

1. European Medicines Agency. Guideline on similar biological medicinal products containing biotechnology-derived proteins as active substance: nonclinical and clinical issues. Published December 18, 2014. Accessed 20 Oct 2021. https://www.ema. europa.eu/en/documents/scientific-guideline/ guideline-similar-biological-medicinal-productscontaining-biotechnology-derived-proteins-active en-2.pdf.

2. US Food and Drug Administration. Guidance for Industry. Scientific considerations in demonstrating biosimilarity to a reference product. Published April 2015. Accessed 20 Oct 2021. https://www.fda.gov/media/82647/download.

3. European Medicines Agency. Biosimilars in the EU. Information guide for healthcare professionals. Accessed 20 Oct 2021. https://www.ema.europa.eu/ en/documents/leaflet/biosimilars-eu-informationguide-healthcare-professionals_en.pdf.

4. US Food and Drug Administration. Clinical pharmacology data to support a demonstration of biosimilarity to a reference product. Published December 2016. Accessed 20 Oct 2021. https:// www.fda.gov/regulatory-information/search-fdaguidance-documents/clinical-pharmacology-datasupport-demonstration-biosimilarity-referenceproduct.

5. Wolff-Holz E, Tiitso K, Vleminckx C, Weise M. Evolution of the EU biosimilar framework: past and future. BioDrugs. 2019;33(6):621-34.

6. Neupogen (filgrastim) injection, for subcutaneous or intravenous use [package insert]. Thousand Oaks, CA: Amgen Inc.; 2021.

7. Neulasta (pegfilgrastim) injection, for subcutaneous use [package insert]. Thousand Oaks, CA: Amgen Inc.; 2021.

8. Arvedson T, O'Kelly J, Yang BB. Design rationale and development approach for pegfilgrastim as a long-acting granulocyte colony-stimulating factor. BioDrugs. 2015;29(3):185-98.

9. Roskos LK, Lum P, Lockbaum P, Schwab G, Yang BB. Pharmacokinetic/pharmacodynamic modeling of pegfilgrastim in healthy subjects. J Clin Pharmacol. 2006;46(7):747-57.

10. Udenyca (pegfilgrastim-cbqv) injection, for subcutaneous use [package insert]. Redwood City, CA: Coherus BioSciences, Inc; 2021.

11. Finck B, Tang H, Civoli F, Hodge J, O'Kelly H, Vexler V. Pharmacokinetic and pharmacodynamic equivalence of pegfilgrastim-cbqv and pegfilgrastim in healthy subjects. Adv Ther. 2020;37(10): 4291-307.

12. Krieckaert C, Rispens T, Wolbink G. Immunogenicity of biological therapeutics: from assay to patient. Curr Opin Rheumatol. 2012;24(3):306-11.

13. Schellekens H. Bioequivalence and the immunogenicity of biopharmaceuticals. Nat Rev Drug Discov. 2002;1(6):457-62.

14. Waller CF, Tiessen RG, Lawrence TE, Shaw A, Liu MS, Sharma R, Baczkowski M, Kothekar MA, Micales CE, Barve A, et al. A pharmacokinetics and 
pharmacodynamics equivalence trial of the proposed pegfilgrastim biosimilar, MYL-1401H, versus reference pegfilgrastim. J Cancer Res Clin Oncol. 2018;144(6):1087-95.

15. Yang BB, Morrow PK, Wu X, Moxness M, Padhi D. Comparison of pharmacokinetics and safety of pegfilgrastim administered by two delivery methods: on-body injector and manual injection with a prefilled syringe. Cancer Chemother Pharmacol. 2015;75(6):1199-206.

16. Chen BM, Su YC, Chang CJ, Burnouf PA, Chuang $\mathrm{KH}$, Chen $\mathrm{CH}$, et al. Measurement of pre-existing IgG and IgM antibodies against polyethylene glycol in healthy individuals. Anal Chem. 2016;88(21): 10661-6.

17. Armstrong JK, Hempel G, Koling S, Chan LS, Fisher T, Meiselman HJ, Garratty G. Antibody against poly(ethylene glycol) adversely affects PEG-asparaginase therapy in acute lymphoblastic leukemia patients. Cancer. 2007;110(1):103-11.

18. Hershfield MS, Ganson NJ, Kelly SJ, Scarlett EL, Jaggers DA, Sundy JS. Induced and pre-existing antipolyethylene glycol antibody in a trial of every 3-week dosing of pegloticase for refractory gout, including in organ transplant recipients. Arthritis Res Ther. 2014;16(2):R63. 